\title{
LOCAL PROPERTIES OF QUASIHYPERBOLIC MAPPINGS IN METRIC SPACES
}

\author{
Xiaojun Huang, Hongjun Liu and Jinsong Liu \\ Chongqing University, College of Mathematics and Statistics \\ Chongqing 401331, P. R. China; hxj@cqu.edu.cn \\ Chongqing University, College of Mathematics and Statistics \\ Chongqing 401331, P. R. China; liuhongjun1121@126.com \\ Chinese Academy of Sciences, HUA Loo-Keng Key Laboratory of Mathematics \\ Beijing 100190, P. R. China; liujsong@math.ac.cn
}

\begin{abstract}
In this paper, we consider Väisälä's problem and obtain that a homeomorphism which is both semi-local $M$-QH and semi-local $\eta$-QS between two suitable metric spaces is an $M_{1}-\mathrm{QH}$ map.
\end{abstract}

\section{Introduction}

During the past few decades, modern geometric function theory of quasisymmetric mappings has been studied from several points of view. Quasisymmetric mappings on the real line were first introduced by Beurling and Ahlfors [1]. They found a way to extend each quasisymmetric self-mapping of the real line to a quasiconformal selfmapping of the upper half-plane. This concept was later promoted by Tukia and Väisälä [8], who introduced and studied quasisymmetric mappings between arbitrary metric spaces. In 1990, based on the idea of quasisymmetry, Väisälä developed a "dimension-free" theory of quasiconformal mappings in infinite-dimensional Banach spaces and obtained many beautiful results. See also [15, 16, 17, 18, 19]. In 1998, Heinonen and Koskela [3] showed that these concepts, quasiconformality and quasisymmetry, are quantitatively equivalent in a large class of metric spaces, which includes Euclidean spaces. Since these two concepts are equivalent, mathematicians show much interest in the research of quasisymmetric mappings between suitable metric spaces.

Following analogous notations and terminologies of $[2,3,9,19]$, we now give the necessary definitions.

Definition 1.1. Let $X, Y$ be two metric spaces. A homeomorphism $f: X \rightarrow$ $Y$ is called $\eta$-quasisymmetric (or briefly $\eta$ - $Q S$ ) if there exists a homeomorphism $\eta:[0, \infty) \rightarrow[0, \infty), \eta(0)=0$, such that

$$
|x-a| \leq t|x-b| \text { implies }|f(x)-f(a)| \leq \eta(t)|f(x)-f(b)|
$$

for each $t>0$ and for each triple $x, a, b$ of points in $X$.

Note that here and hereafter we use the distance notation $|x-y|$ in any metric space.

doi:10.5186/aasfm.2016.4106

2010 Mathematics Subject Classification: Primary 30C65.

Key words: Quasisymmetry, quasihyperbolic metric, quasiconvex metric space.

The first author was supported by NSF of China (No. 11471318) and Natural Science Foundation Project of Chongqing (No. CSTC, 2011BB0055).

The third author was supported by NSFC of China (No. 11471318). 
Definition 1.2. Let $X, Y$ be metric spaces and $G \varsubsetneqq X, G^{\prime} \varsubsetneqq Y$ be two domains, that is, open connected nonempty sets. Suppose that $f: G \rightarrow G^{\prime}$ is a homeomorphism. We say that $f$ is $M$-quasihyperbolic (or briefly $M$ - $Q H$ ) if $f$ is $M$-bilipschitz in quasihyperbolic metrics:

$$
\frac{k_{G}(x, y)}{M} \leq k_{G^{\prime}}(f(x), f(y)) \leq M k_{G}(x, y)
$$

for all $x, y \in G$. For the definition of quasihyperbolic metric, see Section 2 .

A map $f: X \rightarrow Y$ is locally $M$-bilipschitz, $M \geq 1$, if each point $x \in X$ has a neighborhood $U$ such that the restriction $\left.f\right|_{U}$ is $M$-bilipschitz between the quasihyperbolic metrics of $U$ and $f(U)$. In [19], Väisälä proved that such a map is $M^{\prime}$-QH in all Banach spaces.

Theorem 1.3. [19, Theorem 5.16] Let $E$ and $E^{\prime}$ be two Banach spaces and $G \varsubsetneqq$ $E$ be a domain. Suppose that $f: G \rightarrow G^{\prime}$ is a locally $M$-bilipschitz homeomorphism. Then $G^{\prime} \neq E^{\prime}$ and $f$ is $M^{2}-Q H$.

Therefore, Väisälä proposed the following problem.

Problem 1.4. [19, Open problem 13] Suppose that $f: G \rightarrow G^{\prime}$ is a homeomorphism, and there exists $M>1$ such that, for each point $x \in G$, there exists a neighborhood $D(x) \subset G$ such that the restriction map $\left.f\right|_{D(x)}: D(x) \rightarrow f(D(x))$ is $M$-QH. Is $f$ globally $M^{\prime}$-QH with $M^{\prime}=M^{\prime}(M)$ ?

In this paper we shall discuss Väisälä's open problem between two suitable metric spaces. In order to state our result, we give some definitions.

Definition 1.5. Let $X$ be a metric space and $G \varsubsetneqq X$ be a non-empty open set. For any $x \in G$, we denote by $\delta_{G}(x)$ the $\operatorname{distance} \operatorname{dist}(x, X \backslash G)$.

Remark 1.6. From the above definition, it follows that

$$
B\left(x, \delta_{G}(x)\right) \subseteq G
$$

where $B\left(x, \delta_{G}(x)\right)=\left\{y \in X:|y-x|<\delta_{G}(x)\right\}$.

Definition 1.7. A metric space $X$ is said to be dense, if for any two points $x, y \in X$ and two positive real number $r_{1}, r_{2}$ with $|x-y|<r_{1}+r_{2}$, we have $B\left(x, r_{1}\right) \cap$ $B\left(y, r_{2}\right) \neq \emptyset$.

Definition 1.8. Let $X, Y$ be two metric spaces and $G \varsubsetneqq X, G^{\prime} \varsubsetneqq Y$ be two domains. Let $\eta:[0, \infty) \rightarrow[0, \infty), \eta(0)=0$, be a homeomorphism. A homeomorphism $f: G \rightarrow G^{\prime}$ is said to be semi-local $M$ - $Q H$ (resp. semi-local $\eta$ - $Q S$ ), if for each points $x \in G$, the map $\left.f\right|_{B_{x}}: B_{x} \rightarrow f\left(B_{x}\right)$ is $M$-QH (resp. $\eta$-QS). Here $B_{x}=B\left(x, \delta_{G}(x)\right.$ ).

Definition 1.9. A metric space $X$ is said to be proper if every closed ball in $X$ is compact.

Under suitable geometric conditions (see Section 2), we study Väisälä's open problem and obtain an analogous result for metric spaces.

Theorem 1.10. Let $X$ be a $c_{1}$-quasiconvex and dense metric space and let $Y$ be a $c_{2}$-quasiconvex, dense and proper metric space. Let $G \varsubsetneqq X$ and $G^{\prime} \varsubsetneqq Y$ be two domains. Suppose that a homeomorphism $f: G \rightarrow G^{\prime}$ is both semi-local $M-Q H$ and semi-local $\eta$-QS map, where $M>1$ is a constant and $\eta:[0, \infty) \rightarrow[0, \infty), \eta(0)=0$, is a homeomorphism. Then $f$ is an $M_{1}-Q H$ map on $G$ with $M_{1}=M_{1}\left(M, \eta, c_{1}, c_{2}\right)$. 
Example 1.11. Let $X=\left\{\bigcup_{r \in \mathbf{Q}}\{r\} \times \mathbf{R}\right\} \cup\left\{\bigcup_{t \in \mathbf{Q}} \mathbf{R} \times\{t\}\right\}$ be a subset of $\mathbf{R}^{2}$ and the metric $d$ of $X$ be the restriction of Euclidean metric of $\mathbf{R}^{2}$ on $X$. Then $(X, d)$ is a 3-quasiconvex, dense and proper metric space.

We leave the following

Problem 1.12. Can one strengthen the above Theorem 1.10? That is, can one deduce that a homeomorphism $f: G \rightarrow G^{\prime}$ which is locally $M$-QH is a global $M_{1}$-QH map in some suitable metric spaces?

\section{Quasihyperbolic metric}

Let $X$ be a metric space and let

$$
B(x, r)=\{y \in X:|y-x|<r\}, \quad \bar{B}(x, r)=\{y \in X:|y-x| \leq r\}
$$

be the open and close balls with center $x \in X$ and radius $r>0$. Denote

$$
S(x, r)=\{y:|y-x|=r\} .
$$

By a curve we mean any continuous mapping $\gamma:[a, b] \rightarrow X$. The length of $\gamma$ is defined by

$$
l(\gamma)=\sup \left\{\sum_{i=1}^{n}\left|\gamma\left(t_{i}\right)-\gamma\left(t_{i-1}\right)\right|\right\},
$$

where the supremum is taken over all partitions $a=t_{0}<t_{1}<\cdots<t_{n}=b$. The curve is rectifiable if $l(\gamma)<\infty$.

The length function associated with a rectifiable curve $\gamma:[a, b] \rightarrow X$ is $s_{\gamma}:[a, b] \rightarrow$ $[0, l(\gamma)]$, given by $s_{\gamma}(t)=l\left(\left.\gamma\right|_{[a, t]}\right)$. For any rectifiable curve $\gamma:[a, b] \rightarrow X$, there exists a unique curve $\gamma_{s}:[0, l(\gamma)] \rightarrow X$ such that $\gamma=\gamma_{s} \circ s_{\gamma}$. Moreover, $l\left(\left.\gamma_{s}\right|_{[0, t]}\right)=t$ for every $t \in[0, l(\gamma)]$. The curve $\gamma_{s}$ is called the arc length parametrization of $\gamma$.

If $\gamma$ is a rectifiable curve in $X$, then the line integral over $\gamma$ of each nonnegative Borel function $\varrho: X \rightarrow[0, \infty]$ is given by

$$
\int_{\gamma} \varrho d s=\int_{0}^{l(\gamma)} \varrho \circ \gamma_{s}(t) d t .
$$

Definition 2.1. Let $\gamma$ be a rectifiable curve in a domain $G \varsubsetneqq X$. The quasihyperbolic length of $\gamma$ in $G$ is

$$
l_{\mathrm{qh}}(\gamma)=\int_{\gamma} \frac{d s}{\delta_{G}(x)}
$$

The quasihyperbolic distance between $x, y \in G$ is defined by

$$
k_{G}(x, y)=\inf _{\gamma} l_{\mathrm{qh}}(\gamma)
$$

where $\gamma$ runs over all rectifiable curves in $G$ joining $x$ and $y$. If there is no rectifiable curve in $G$ joining $x$ and $y$, then we define

$$
k_{G}(x, y)=+\infty
$$

Definition 2.2. Let $X$ be a metric space. An open set $D$ of $X$ is said to be rectifiably connected if, for any two points $x, y \in D$, there exists a rectifiable curve in $D$ joining $x$ and $y$.

If $G \varsubsetneqq X$ is a rectifiably connected open set, it is clear that $k_{G}(x, y)<\infty$ for any two points $x, y \in G$. Thus $k_{G}$ is a metric in $G$, and we call it the quasihyperbolic metric of $G$. 
Definition 2.3. For $c \geq 1$, a metric space $X$ is c-quasiconvex if each pair of points $x, y \in X$ can be joined by a curve $\gamma$ with length $l(\gamma) \leq c|x-y|$.

Observation 2.4. If $X$ is a c-quasiconvex metric space, then any domain $G \varsubsetneqq X$ is rectifiably connected.

Proof. Choose $x_{0} \in G$ and define

$$
O_{x_{0}}=\left\{y \in G \text { : there exists a rectifiable curve in } G \text { joining } x_{0} \text { and } y\right\} .
$$

It is clear that $x_{0} \in O_{x_{0}}$.

We claim that $O_{x_{0}}$ and $G \backslash O_{x_{0}}$ are both open in $G$. Noticing that $G$ is open, for any $y_{0} \in O_{x_{0}}$, there exists a $r>0$ such that $B\left(y_{0}, r\right) \subseteq G$. For any $z \in B\left(y_{0}, r / c\right)$, by using the definition of quasiconvexity, it follows that there exists a rectifiably curve $\gamma$ joining $y_{0}$ and $z$ with

$$
l(\gamma) \leq c\left|y_{0}-z\right|<r
$$

which implies $\gamma \subseteq G$. Thus,

$$
B\left(y_{0}, r / c\right) \subseteq O_{x_{0}}
$$

which shows that $O_{x_{0}}$ is open in $G$.

With a similar argument, we can deduce that $G \backslash O_{x_{0}}$ is also open in $G$. Since $G$ is connected, we have $O_{x_{0}}=G$. Hence $G$ is rectifiably connected.

Theorem 2.5. Let $X$ be a c-quasiconvex metric space and let $G \varsubsetneqq X$ be a domain. Then

(i) for each $x, y \in G$,

$$
|x-y| \leq\left(e^{k_{G}(x, y)}-1\right) \delta_{G}(x)
$$

(ii) if $z \in G, 0<t<1$, and $x, y \in \bar{B}\left(z, \frac{t \delta_{G}(z)}{4 c}\right)$, then

$$
\frac{1}{1+2 t} \frac{|x-y|}{\delta_{G}(z)} \leq k_{G}(x, y) \leq \frac{c}{1-t} \frac{|x-y|}{\delta_{G}(z)} .
$$

Proof. (i) By Observation 2.4, we know that $G$ is rectifiably connected. For any rectifiable curve $\gamma$ joining $x, y \in G$, let $\gamma_{s}:[0, L] \rightarrow G$ be the arc length parametrization of $\gamma$ with $\gamma_{s}(0)=x$. We have, for each $t \in[0, L]$,

$$
\delta_{G}\left(\gamma_{s}(t)\right) \leq \delta_{G}(x)+\left|\gamma_{s}(t)-x\right| \leq \delta_{G}(x)+l\left(\left.\gamma_{s}\right|_{[0, t]}\right)=\delta_{G}(x)+t .
$$

Hence

$$
l_{\mathrm{qh}}(\gamma) \geq \int_{0}^{L} \frac{d t}{\delta_{G}(x)+t} \geq \ln \left(1+\frac{|x-y|}{\delta_{G}(x)}\right) .
$$

The inequality (1) is a consequence of the definition of $k_{G}(x, y)$ and the above inequality.

(ii) Suppose that $x, y \in \bar{B}\left(z, \frac{t \delta_{G}(z)}{4 c}\right)$. Since $X$ is a $c$-quasiconvex metric space, there exists a rectifiable curve $\gamma$ in $X$ joining $x$ to $y$ with $l(\gamma) \leq c|x-y|$.

For any $u \in \gamma$, it is clear that

$$
\begin{aligned}
|u-z| & \leq|u-x|+|x-z| \leq l(\gamma)+t \delta_{G}(z) /(4 c) \\
& \leq c|x-y|+t \delta_{G}(z) /(4 c) \leq((2 c+1) /(4 c)) \cdot t \cdot \delta_{G}(z) \quad(\text { since } c \geq 1) \\
& <t \delta_{G}(z),
\end{aligned}
$$

which implies that

$$
\gamma \subseteq B\left(z, t \delta_{G}(z)\right) \subseteq B\left(z, \delta_{G}(z)\right) \subseteq G
$$


Furthermore, for each $u \in \gamma$, we have

$$
\delta_{G}(u) \geq \delta_{G}(z)-|u-z| \geq(1-t) \delta_{G}(z) .
$$

Let $L=l(\gamma)$ and let $\gamma_{s}:[0, L] \rightarrow \gamma$ be the arc length parametrization of $\gamma$. Hence

$$
k_{G}(x, y) \leq \int_{0}^{L} \frac{d r}{\delta_{G}\left(\gamma_{s}(r)\right)} \leq \frac{L}{(1-t) \delta_{G}(z)} \leq \frac{c}{1-t} \frac{|x-y|}{\delta_{G}(z)}
$$

which shows that the right-hand side inequality of (2) is true.

Now we are ready to verify the left-hand side inequality of (2). Since $G$ is rectifiably connected, the set of rectifiable curves joining $x$ and $y$ is not empty. Assume that $\gamma:[0, L] \rightarrow G$ is any rectifiable curve joining $x, y \in G$ which is parametrized by its arc length. Then we divide the proof into two parts:

Case 1. $\gamma \subseteq \bar{B}\left(z, 2 t \delta_{G}(z)\right)$. In this case, for all $u \in \gamma$,

$$
\delta_{G}(u) \leq|u-z|+\delta_{G}(z) \leq(1+2 t) \delta_{G}(z) .
$$

Therefore, it follows that

$$
l_{\mathrm{qh}}(\gamma)=\int_{0}^{L} \frac{d r}{\delta_{G}\left(\gamma_{s}(r)\right)} \geq \frac{l(\gamma)}{1+2 t} \frac{1}{\delta_{G}(z)} \geq \frac{|x-y|}{(1+2 t) \delta_{G}(z)} .
$$

Case 2. $\gamma \nsubseteq \bar{B}\left(z, 2 t \delta_{G}(z)\right)$. From the connectedness of $\gamma$, it follows that $\gamma$ has a sub-curve $\gamma_{1} \subseteq \bar{B}\left(z, 2 t \delta_{G}(z)\right)$ joining the spheres $S\left(z, t \delta_{G}(z)\right)$ and $S\left(z, 2 t \delta_{G}(z)\right)$ which implies

$$
l\left(\gamma_{1}\right) \geq t \delta_{G}(z)
$$

Since $|x-y| \leq \frac{t \delta_{G}(x)}{2 c}$, it follows that

$$
l\left(\gamma_{1}\right) \geq 2 c|x-y| .
$$

For any $u \in \gamma_{1}$, we have $\delta_{G}(u) \leq(1+2 t) \delta_{G}(z)$. Thus, we again obtain (3).

Hence, the inequality (2) is obtained.

Theorem 2.6. Let $X$ be a c-quasiconvex metric space and $G \varsubsetneqq X$ be a domain. Suppose that $x, y \in G$ and either $|x-y| \leq \frac{\delta_{G}(x)}{8 c}$ or $k_{G}(x, y) \leq \frac{1}{4}$. Then

$$
\frac{1}{2} \frac{|x-y|}{\delta_{G}(x)} \leq k_{G}(x, y) \leq 2 c \frac{|x-y|}{\delta_{G}(x)} .
$$

Proof. If $|x-y| \leq \delta_{G}(x) /(8 c)$, then (4) is a consequence of Theorem 2.5 with $t=1 / 2$. Thus we may assume that $|x-y|>\frac{\delta_{G}(x)}{8 c}$ and $k_{G}(x, y) \leq \frac{1}{4}$. From this assumption, it is easily be seen that

$$
k_{G}(x, y) \leq 2 c \frac{|x-y|}{\delta_{G}(x)},
$$

that is, the right-hand side inequality of (4) holds.

We are now to prove the left-hand side inequality of (4). Since $e^{r}-1 \leq 2 r$ for $r \in(0,1 / 4]$, it follows from the inequality (1) in Theorem 2.5 that

$$
\frac{|x-y|}{\delta_{G}(x)} \leq e^{k_{G}(x, y)}-1 \leq 2 k_{G}(x, y),
$$

which implies the left-hand side inequality of (4) is also true. Hence, Theorem 2.6 is proved. 
Theorem 2.7. Let $X$ be a $c$-quasiconvex metric space and $G \varsubsetneqq X$ be a domain. Let $\gamma$ be a rectifiable path in $G$ and let $l_{k_{G}}(\gamma)$ denote the length of $\gamma$ in the metric space $\left(G, k_{G}\right)$. Then

(i) $l_{k_{G}}(\gamma)=l_{\mathrm{qh}}(\gamma)$;

(ii) the metric space $\left(G, k_{G}\right)$ is a 2-quasiconvex metric space.

Proof. (i) Let $\gamma_{s}$ be the arc length parametrization of $\gamma$ and $L$ be the length of $\gamma$. Let $0=t_{0}<t_{1}<\cdots<t_{n}=L$ be a partition of $[0, L]$. Then

$$
\sum_{j=1}^{n} k_{G}\left(\gamma_{s}\left(t_{j-1}\right), \gamma_{s}\left(t_{j}\right)\right) \leq \sum_{j=1}^{n} l_{\mathrm{qh}}\left(\left.\gamma_{s}\right|_{\left[t_{j-1}, t_{j}\right]}\right) \leq l_{\mathrm{qh}}(\gamma) .
$$

Hence $l_{k_{G}}(\gamma) \leq l_{\mathrm{qh}}(\gamma)$.

Now we are turn to prove $l_{k_{G}}(\gamma) \geq l_{\mathrm{qh}}(\gamma)$. By definition of $l_{\mathrm{qh}}(\gamma)$, it follows that

$$
l_{\mathrm{qh}}(\gamma)=\int_{0}^{L} g(t) d t
$$

where $g(t)=1 / \delta_{G}\left(\gamma_{s}(t)\right)$. Choose $0<\epsilon<1 / 2$. By definition of Riemann integral $\int_{0}^{L} g(t) d t$ and the compact proposition of $\gamma$, we can obtain the following fact:

Fact. There exists a partition $P: 0=t_{0}<t_{1}<\cdots<t_{n}=L$ of $[0, L]$ such that, for $x_{i}=\gamma_{s}\left(t_{i}\right), \gamma_{i}=\left.\gamma_{s}\right|_{\left[t_{i-1}, t_{i}\right]}$ and $1 \leq i \leq n$, we have

$$
l_{\mathrm{qh}}(\gamma) \leq \sum_{i=1}^{n} g\left(t_{i}\right)\left(t_{i}-t_{i-1}\right)+\epsilon \quad \text { and } \quad \gamma_{i} \subseteq B\left(x_{i}, \frac{\epsilon \cdot \delta_{G}\left(x_{i}\right)}{8 c}\right) .
$$

The verification of this fact is left to the reader.

For each $1 \leq i \leq n$, we choose successive points $x_{i-1}=x_{i, 0}, x_{i, 1}, \cdots, x_{i, n_{i}}=x_{i}$ of $\gamma_{i}$ such that

$$
l\left(\gamma_{i}\right) \leq \sum_{j=1}^{n_{i}}\left|x_{i, j-1}-x_{i, j}\right|+\epsilon / n .
$$

With the aid of the estimate (2) in Theorem 2.5, we get that, for all $1 \leq i \leq n$ and $1 \leq j \leq n_{i}$,

$$
\frac{\left|x_{i, j-1}-x_{i, j}\right|}{\delta_{G}\left(x_{i}\right)} \leq(1+2 \epsilon) \cdot k_{G}\left(x_{i, j-1}, x_{i, j}\right) .
$$

Since $t_{i}-t_{i-1}=l\left(\gamma_{i}\right)$ and $g\left(t_{i}\right)=1 / \delta_{G}\left(x_{i}\right)$, the above estimates imply

$$
l_{\mathrm{qh}}(\gamma) \leq(1+2 \epsilon) \sum_{i} \sum_{j} k_{G}\left(x_{i, j-1}, x_{i, j}\right)+2 \epsilon .
$$

Notice that the double sum is at most $l_{k_{G}}(\gamma)$. Since $\epsilon$ is arbitrary, this yields the desired inequality.

(ii) Obviously, we have nothing to do if the cardinal number $|G|$ of $G$ is less than 2. So we suppose that $|G| \geq 2$. Let $a \neq b$ be two points in $G$.

According to Observation 2.4 and the definition of $k_{G}(a, b)$, it follows that there exists a path $\gamma$ joining $a$ and $b$ with $l_{\mathrm{qh}}(\gamma)<2 k_{G}(a, b)$. By (i), we get $l_{k_{G}}(\gamma)<$ $2 k_{G}(a, b)$, which implies that $\left(G, k_{G}\right)$ is 2-quasiconvex. 


\section{Quasihyperbolic and fully quasihyperbolic maps}

Definition 3.1. Let $f: X \rightarrow Y$ be a map between metric spaces and let $x$ be a non-isolated point of $X$. We write

$$
L(x, f)=\limsup _{y \rightarrow x} \frac{|f(y)-f(x)|}{|y-x|}, \quad l(x, f)=\liminf _{y \rightarrow x} \frac{|f(y)-f(x)|}{|y-x|} .
$$

Then $0 \leq l(x, f) \leq L(x, f) \leq \infty$. The number $L(x, f)$ is the maximal stretching of $f$ at $x$, and $l(x, f)$ is the minimal stretching. In the case when $X=G$ and $Y=G^{\prime}$ with the QH metrics, we always use the notations $L_{k}(x, f), l_{k}(x, f)$.

Fact 3.2. (i) Suppose that $f: X \rightarrow Y$ is a homeomorphism and $x$ is a nonisolated point of $X$. Then

$$
L(x, f)=\frac{1}{l\left(f(x), f^{-1}\right)}, \quad l(x, f)=\frac{1}{L\left(f(x), f^{-1}\right)},
$$

where we use the convention that $1 / 0=\infty, 1 / \infty=0$.

(ii) Suppose that $f: X \rightarrow Y$ and $g: Y \rightarrow Z$ are continuous, and that the points $x$ and $f(x)$ are non-isolated points of $X$ and $Y$, respectively. Then

$$
L(x, g \circ f) \leq L(x, f) \cdot L(f(x), g), \quad l(x, g \circ f) \geq l(x, f) \cdot l(f(x), g),
$$

provided that the products are not of the form $0 \cdot \infty$ or $\infty \cdot 0$.

Theorem 3.3. Let $X$ be a $c_{1}$-quasiconvex metric space and $Y$ be a $c_{2}$-quasiconvex metric space, and let $G \varsubsetneqq X$ and $G^{\prime} \varsubsetneqq Y$ be two domains. If $f: G \rightarrow G^{\prime}$ is a homeomorphism, then

$$
\frac{1}{c_{1}} \frac{L(x, f) \cdot \delta_{G}(x)}{\delta_{G^{\prime}}(f(x))} \leq L_{k}(x, f) \leq \frac{c_{2} L(x, f) \cdot \delta_{G}(x)}{\delta_{G^{\prime}}(f(x))}
$$

and

$$
\frac{1}{c_{1}} \frac{l(x, f) \cdot \delta_{G}(x)}{\delta_{G^{\prime}}(f(x))} \leq l_{k}(x, f) \leq \frac{c_{2} l(x, f) \cdot \delta_{G}(x)}{\delta_{G^{\prime}}(f(x))}
$$

for all $x \in G$.

Proof. Let $d_{G}$ (resp. $d_{G^{\prime}}$ ) denote the metric of $G$ (resp. $G^{\prime}$ ) be the restriction of Euclidean metric of $X$ (resp. $Y$ ) on $G$ (resp. $\left.G^{\prime}\right)$, and $k_{G}\left(\right.$ resp. $\left.k_{G^{\prime}}\right)$ denote the quasihyperbolic metric of $G$ (resp. $\left.G^{\prime}\right)$, and let $i:\left(G, k_{G}\right) \rightarrow\left(G, d_{G}\right)$ and $j:\left(G^{\prime}, k_{G^{\prime}}\right) \rightarrow\left(G^{\prime}, d_{G^{\prime}}\right)$ be the identity maps. By Theorem 2.5 it follows that

$$
\frac{1}{c_{1}} \delta_{G}(x) \leq L(x, i) \leq \delta_{G}(x), \quad \delta_{G}(x)^{-1} \leq L\left(x, i^{-1}\right) \leq c_{1} \delta_{G}(x)^{-1}
$$

and

$$
\begin{aligned}
& \frac{1}{c_{2}} \delta_{G^{\prime}}(f(x)) \leq L(f(x), j) \leq \delta_{G^{\prime}}(f(x)) \\
& \delta_{G^{\prime}}(f(x))^{-1} \leq L\left(f(x), j^{-1}\right) \leq c_{2} \delta_{G^{\prime}}(f(x))^{-1} .
\end{aligned}
$$


Consider $f$ as a map $f:\left(G, d_{G}\right) \rightarrow\left(G^{\prime}, d_{G^{\prime}}\right)$, and let $f_{k}:\left(G, k_{G}\right) \rightarrow\left(G^{\prime}, k_{G^{\prime}}\right)$ be the map with the same values of $f$. Since $f_{k}=j^{-1} \circ f \circ i$ and $f=j \circ f_{k} \circ i^{-1}$, we have

$$
\begin{aligned}
L_{k}(x, f) & =L\left(x, f_{k}\right) \leq L(x, i) \cdot L(x, f) \cdot L\left(f(x), j^{-1}\right) \leq \frac{c_{2} L(x, f) \delta_{G}(x)}{\delta_{G^{\prime}}(f(x))}, \\
L(x, f) & =L\left(x, j \circ f_{k} \circ i^{-1}\right) \leq L\left(x, i^{-1}\right) \cdot L\left(x, f_{k}\right) \cdot L(f(x), j) \\
& \leq \frac{c_{1} L\left(x, f_{k}\right) \cdot \delta_{G^{\prime}}(f(x))}{\delta_{G}(x)}=\frac{c_{1} L_{k}(x, f) \cdot \delta_{G^{\prime}}(f(x))}{\delta_{G}(x)} .
\end{aligned}
$$

Therefore, (5) holds. The proof of (6) is similar.

Lemma 3.4. [15, Lemma 4.4] Let $f: X \rightarrow Y$ be a homeomorphism. If $f$ is $M$-Lipschitz, then $L(x, f) \leq M$ for all non-isolated points $x \in X$. Conversely, if $X$ is c-quasiconvex and if $L(x, f) \leq M$ for all $x \in X$, then $f$ is $c M$-Lipschitz.

Theorem 3.5. Let $X$ be a $c_{1}$-quasiconvex metric space and let $Y$ be a $c_{2}$ quasiconvex metric space. Suppose that $G \varsubsetneqq X$ and $G^{\prime} \varsubsetneqq Y$ are two domains, and that $f: G \rightarrow G^{\prime}$ is a homeomorphism.

(i) If $f$ is $M-Q H$, then, for all $x \in G$,

$$
L(x, f) \cdot \delta_{G}(x) \leq c_{1} M \cdot \delta_{G^{\prime}}(f(x)), \quad L\left(f(x), f^{-1}\right) \cdot \delta_{G^{\prime}}(f(x)) \leq c_{2} M \cdot \delta_{G}(x) .
$$

(ii) Conversely, if, for all $x \in G$,

$$
L(x, f) \cdot \delta_{G}(x) \leq c_{1} M \cdot \delta_{G^{\prime}}(f(x)), \quad L\left(f(x), f^{-1}\right) \cdot \delta_{G^{\prime}}(f(x)) \leq c_{2} M \cdot \delta_{G}(x),
$$

then $f$ is $2 c_{1} c_{2} M-Q H$.

Proof. We first prove (i). By symmetry it suffices to prove that

$$
L(x, f) \cdot \delta_{G}(x) \leq c_{1} M \cdot \delta_{G^{\prime}}(f(x))
$$

for all $x, y \in G$. Since $f$ is $M$-QH, it follows immediately from Lemma 3.4 that

$$
L_{k}(x, f) \leq M
$$

Thus, by combing Theorem 3.3, we get

$$
\frac{1}{c_{1}} \frac{L(x, f) \cdot \delta_{G}(x)}{\delta_{G^{\prime}}(f(x))} \leq L_{k}(x, f) \leq M .
$$

Hence the proof of (i) is complete.

Now we are ready to prove (ii). By Theorem 2.7 , we can deduce that both $\left(G, k_{G}\right)$ and $\left(G^{\prime}, k_{G^{\prime}}\right)$ are 2-quasiconvex metric spaces. Since

$$
L(x, f) \cdot \delta_{G}(x) \leq c_{1} M \cdot \delta_{G^{\prime}}(f(x)),
$$

it follows from the Theorem 3.3 that

$$
L_{k}(x, f) \leq c_{1} c_{2} M
$$

Therefore, according to (7) and Lemma 3.4, we obtain that $f$ is $2 c_{1} c_{2} M$-Lipschitz in the $\mathrm{QH}$ metric. By using the same argument, we deduce that $f^{-1}$ is $2 c_{1} c_{2} M$-Lipschitz in the $\mathrm{QH}$ metric.

Hence, $f$ is $2 c_{1} c_{2} M$-bilipschitz in the $\mathrm{QH}$ metric. 
Definition 3.6. Let $X, Y$ be two metric spaces and $G \varsubsetneqq X, G^{\prime} \varsubsetneqq Y$ be two domains. A homeomorphism $f: G \rightarrow G^{\prime}$ is called fully $M^{\prime}-Q H$, if for any sub-domain $D \subseteq G$, the map $\left.f\right|_{D}: D \rightarrow D^{\prime}=f(D)$ is $M^{\prime}-\mathrm{QH}$ with respect to the $\mathrm{QH}$ metric of $D$. That is,

$$
\frac{1}{M^{\prime}} k_{D}(x, y) \leq k_{D^{\prime}}(f(x), f(y)) \leq M^{\prime} k_{D}(x, y)
$$

In what follows, we get the following result which will be used later in this paper.

Theorem 3.7. Let $X$ be a $c_{1}$-quasiconvex metric space and let $Y$ be a $c_{2}$ quasiconvex metric space. Suppose that $G \varsubsetneqq X$ and $G^{\prime} \varsubsetneqq Y$ are two domains and $f: G \rightarrow G^{\prime}$ is $M-Q H$. Then $f$ is fully $M_{1}-Q H$ with $M_{1}=16 c_{1} c_{2} M^{2} \cdot \max \left\{c_{1}, c_{2}\right\}$.

Proof. Let $D \subset G$ be any sub-domain of $G$ and $D^{\prime}=f(D)$. For any given point $x \in D$, we claim that

$$
\frac{1}{8 c_{1} M} \frac{\delta_{G}(x)}{\delta_{G^{\prime}}(f(x))} \leq \frac{\delta_{D}(x)}{\delta_{D^{\prime}}(f(x))} \leq 8 c_{2} M \frac{\delta_{G}(x)}{\delta_{G^{\prime}}(f(x))} .
$$

In what follows, we only prove the right-hand side inequality of (8). The proof of the left-hand side inequality of (8) uses the same argument for $f^{-1}$.

Denote by

$$
\alpha=\frac{\delta_{D}(x)}{\delta_{D^{\prime}}(f(x))} .
$$

We consider two cases:

Case 1. $\delta_{D^{\prime}}(f(x)) \geq \delta_{G^{\prime}}(f(x)) /\left(8 c_{2} M\right)$. Then

$$
\alpha \leq 8 c_{2} M \cdot \frac{\delta_{D}(x)}{\delta_{G^{\prime}}(f(x))} \leq 8 c_{2} M \cdot \frac{\delta_{G}(x)}{\delta_{G^{\prime}}(f(x))} .
$$

Thus, the right-hand side inequality of (8) is obtained.

Case 2. $\delta_{D^{\prime}}(f(x))<\delta_{G^{\prime}}(f(x)) /\left(8 c_{2} M\right)$. Choose a sufficiently small number $\varepsilon$ with

$$
0<\varepsilon<\frac{\delta_{G^{\prime}}(f(x))}{8 c_{2} M}-\delta_{D^{\prime}}(f(x)) .
$$

The definition of $\delta_{D^{\prime}}(f(x))$ allows us to choose a point $y^{\prime} \in Y \backslash D^{\prime}$ such that

$$
\left|f(x)-y^{\prime}\right|<\delta_{D^{\prime}}(f(x))+\varepsilon<\frac{\delta_{G^{\prime}}(f(x))}{8 c_{2} M} .
$$

Thus, we have

$$
\delta_{G^{\prime}}\left(y^{\prime}\right) \geq \delta_{G^{\prime}}(f(x))-\left|f(x)-y^{\prime}\right|>\left(1-\frac{1}{8 c_{2} M}\right) \delta_{G^{\prime}}(f(x))>0 .
$$

Hence, it follows that $y^{\prime} \in G^{\prime}$.

Setting $y=f^{-1}\left(y^{\prime}\right)$. It is clear that

$$
y \in G \backslash D .
$$

From (9) we know that

$$
\frac{\left|f(x)-y^{\prime}\right|}{\delta_{G^{\prime}}(f(x))}<\frac{1}{8 c_{2} M}<\frac{1}{8 c_{2}},
$$

and by use of Theorem 2.6 we obtain from (10) that

$$
k_{G^{\prime}}\left(f(x), y^{\prime}\right) \leq 2 c_{2} \cdot \frac{\left|f(x)-y^{\prime}\right|}{\delta_{G^{\prime}}(f(x))}<\frac{1}{4 M} .
$$


Since $f$ is $M-\mathrm{QH}$, then

$$
k_{G}(x, y) \leq M k_{G^{\prime}}\left(f(x), y^{\prime}\right)<\frac{1}{4} .
$$

Applying Theorem 2.6, we get that

$$
\begin{aligned}
\delta_{D}(x) & \leq|x-y| \quad(\text { since } y \notin D) \\
& \leq 2 k_{G}(x, y) \cdot \delta_{G}(x) \\
& \leq 2 M \cdot k_{G^{\prime}}\left(f(x), y^{\prime}\right) \cdot \delta_{G}(x) \quad(\text { since } f \text { is } M-\mathrm{QH}) \\
& \leq 4 c_{2} M \cdot \frac{\delta_{G}(x)}{\delta_{G^{\prime}}(f(x))} \cdot\left|f(x)-y^{\prime}\right| \quad(\text { by }(11)) \\
& <4 c_{2} M \cdot \frac{\delta_{G}(x)}{\delta_{G^{\prime}}(f(x))}\left(\delta_{D^{\prime}}(f(x))+\varepsilon\right) . \quad(\text { by }(9))
\end{aligned}
$$

As $\varepsilon \rightarrow 0,(12)$ implies that

$$
\alpha \leq 4 c_{2} M \cdot \frac{\delta_{G}(x)}{\delta_{G^{\prime}}(f(x))} .
$$

Therefore, the right-hand side inequality of (8) holds.

Since $f$ is $M$-QH, from (i) of Theorem 3.5, we deduce that

$$
L(x, f) \cdot \frac{\delta_{G}(x)}{\delta_{G^{\prime}}(f(x))} \leq c_{1} M .
$$

Thus, by combing (8) with (13), we get that

$$
L(x, f) \cdot \frac{\delta_{D}(x)}{\delta_{D^{\prime}}(f(x))} \leq 8 c_{2} M \cdot L(x, f) \cdot \frac{\delta_{G}(x)}{\delta_{G^{\prime}}(f(x))} \leq 8 c_{1} c_{2} M^{2} .
$$

Therefore, by the inequality (ii) of Theorem 3.5, we deduce that $\left.f\right|_{D}: D \rightarrow D^{\prime}$ is $M_{1}-\mathrm{QH}$ with respect to $\mathrm{QH}$ metric of $D$. Here

$$
M_{1}=16 c_{1} c_{2} M^{2} \cdot \max \left\{c_{1}, c_{2}\right\} \text {. }
$$

\section{Main lemmas}

Lemma 4.1. Let $X$ be a c-quasiconvex metric space, and let $G \varsubsetneqq X$ be a domain. Suppose that $x \in G$ and $0<s<1 / c$. If $|x-y| \leq s \delta_{G}(x)$, then

$$
k_{B_{x}}(x, y) \leq \frac{c}{1-s c} \ln \left(1+\frac{|x-y|}{\delta_{G}(x)}\right)
$$

where $B_{x}=B\left(x, \delta_{G}(x)\right)$.

Proof. From the definition of quasiconvexity, it follows that there exists a rectifiable arc $\gamma$ joining $x$ and $y$ in $X$ with length $l(\gamma) \leq c|x-y|$. Let $\gamma_{s}:[0, l(\gamma)] \rightarrow \gamma$ be the arc length parametrization of $\gamma$ with $\gamma_{s}(0)=x$. For each $t \in[0, l(\gamma)]$, we have $\left|\gamma_{s}(t)-x\right| \leq l(\gamma)$ and

$$
l(\gamma) \leq c \cdot|x-y| \leq s \cdot c \cdot \delta_{G}(x)<\delta_{G}(x) .
$$

Hence, by the definition of $\delta_{G}(x)$, we get

$$
\gamma \subseteq B_{x} \subseteq G
$$

Since $X \backslash B_{x}=\left\{y \in X:|y-x| \geq \delta_{G}(x)\right\}$, it follows that

$$
\operatorname{dist}\left(x, X \backslash B_{x}\right) \geq \delta_{G}(x) \text {. }
$$


Thus,

$$
\begin{aligned}
\delta_{G}(x) & \leq \operatorname{dist}\left(x, X \backslash B_{x}\right) \leq \operatorname{dist}(x, X \backslash G) \quad\left(\text { since } B_{x} \subseteq G\right) \\
& =\delta_{G}(x) .
\end{aligned}
$$

Therefore, since $\delta_{B_{x}}(x)=\operatorname{dist}\left(x, X \backslash B_{x}\right)$, we obtain

$$
\delta_{B_{x}}(x)=\delta_{G}(x) .
$$

Furthermore,

$$
\begin{aligned}
\delta_{G}(x) & =\delta_{B_{x}}(x)=\operatorname{dist}\left(x, X \backslash B_{x}\right) \leq\left|\gamma_{s}(t)-x\right|+\operatorname{dist}\left(\gamma_{s}(t), X \backslash B_{x}\right) \\
& =\left|\gamma_{s}(t)-\gamma_{s}(0)\right|+\operatorname{dist}\left(\gamma_{s}(t), X \backslash B_{x}\right) \leq t+\delta_{B_{x}}\left(\gamma_{s}(t)\right) .
\end{aligned}
$$

For $t \in[0, l(\gamma)]$, noticing that $\gamma_{s}$ is an arc length parametrization, we have

$$
\left.t=l\left(\left.\gamma_{s}\right|_{[0, t]}\right) \leq l(\gamma)<\delta_{G}(x) . \quad \text { (because of the inequality }(14)\right)
$$

Thus, by (16), it follows that

$$
\frac{1}{\delta_{B_{x}}\left(\gamma_{s}(t)\right)} \leq \frac{1}{\delta_{G}(x)-t}
$$

Hence, from $(15),(17)$ and the definition of $k_{B_{x}}(x, y)$, it follows that

$$
\begin{aligned}
k_{B_{x}}(x, y) & \leq \int_{0}^{l(\gamma)} \frac{d t}{\delta_{B_{x}}\left(\gamma_{s}(t)\right)} \leq \int_{0}^{l(\gamma)} \frac{d t}{\delta_{G}(x)-t}=\ln \left(1+\frac{l(\gamma)}{\delta_{G}(x)-l(\gamma)}\right) \\
& \leq \ln \left(1+\frac{c|x-y|}{\delta_{G}(x)-c|x-y|}\right) \leq \ln \left(1+\frac{c}{1-s c} \cdot \frac{|x-y|}{\delta_{G}(x)}\right) \\
& \leq \frac{c}{1-s c} \ln \left(1+\frac{|x-y|}{\delta_{G}(x)}\right) . \quad \text { (by Bernoulli's inequality) }
\end{aligned}
$$

Here, the statement of Bernoulli's inequality please refer to Appendix. This completes the proof of Lemma 4.1.

Lemma 4.2. Let $X$ be a $c_{1}$-quasiconvex and dense metric space and let $Y$ be a $c_{2}$-quasiconvex, dense and proper metric space. Suppose that $G \varsubsetneqq X, G^{\prime} \varsubsetneqq Y$ are two domains, $x \in G$ is a given point and $f: G \rightarrow G^{\prime}$ is a homeomorphism. Denote by $D$ the open ball $B\left(x, \delta_{G}(x)\right)$ and $D^{\prime}$ the image of $D$ under the homeomorphism $f$. If $\left.f\right|_{D}: D \rightarrow D^{\prime}$ is $\eta$-QS, then

$$
\delta_{D^{\prime}}(f(x)) \geq q \cdot \delta_{G^{\prime}}(f(x)) .
$$

Here $q=\min \left\{1 / 2,2 /\left(3 \eta^{2}(2)\right)\right\}$.

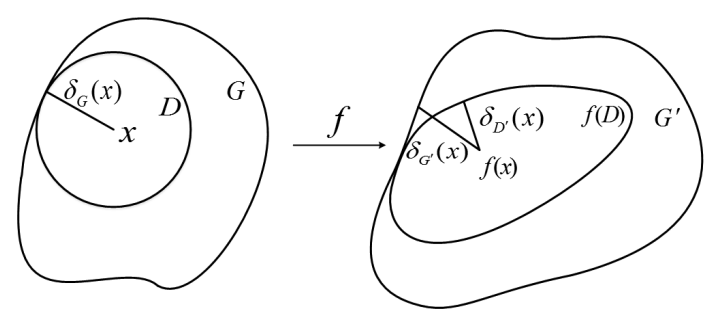

Figure 1. The set $D$ and the set $f(D)$.

To prove the lemma, we need several propositions. The proofs given here depend on a simple geometric intuition as in Figure 1. 
Proposition 4.1. Let $X, Y, G, G^{\prime}, f, D, D^{\prime}$ be as in Lemma 4.2. Let $0<\tau<1$. Then the image of $D$ under a homeomorphism $f$ can not be contained in a ball, with the center $f(x)$, whose radius is less than $\delta_{G^{\prime}}(f(x))$, that is

$$
D^{\prime} \nsubseteq B\left(f(x), \tau \cdot \delta_{G^{\prime}}(f(x))\right) .
$$

Proof. If the statement was false, then

$$
D^{\prime} \subseteq B\left(f(x), \tau \cdot \delta_{G^{\prime}}(f(x))\right) .
$$

First, we need to verify that there exist a sequences $\left\{x_{n}\right\}$ in $D$ which has an accumulation point in $X \backslash G$.

By definition of $\delta_{G}(x)$, it is clear that there exists a sequence of points $\left\{y_{n}\right\} \subseteq$ $X \backslash G$ such that

$$
\delta_{G}(x) \leq\left|y_{n}-x\right|<\delta_{G}(x)+1 / n .
$$

Thanks to the metric space $X$ being dense and the above inequality, it follows that

$$
B\left(y_{n}, \frac{1}{n}\right) \cap B\left(x, \delta_{G}(x)\right) \neq \emptyset .
$$

Choosing the points $\left\{x_{n}\right\}$ with

$$
x_{n} \in B\left(y_{n}, \frac{1}{n}\right) \cap B\left(x, \delta_{G}(x)\right),
$$

it is evident that

$$
\lim _{n \rightarrow \infty} \operatorname{dist}\left(x_{n}, X \backslash G\right)=0 .
$$

The next thing to do in the proof is to show that the sequence of points $\left\{x_{n}\right\}$ has a cluster point in $G$. Due to (18) and $x_{n} \in D$, it is obvious that

$$
f\left(x_{n}\right) \in D^{\prime} \subseteq B\left(f(x), \tau \cdot \delta_{G^{\prime}}(f(x))\right) .
$$

Noting $Y$ being proper, it follows that the close ball $\bar{B}\left(f(x), \tau \cdot \delta_{G^{\prime}}(f(x))\right)$ is compact. This fact implies that the sequence of points $\left\{f\left(x_{n}\right)\right\}$ has an accumulation point in $\bar{B}\left(f(x), \tau \cdot \delta_{G^{\prime}}(f(x))\right)$.

Without loss of generality, we may assume that $f\left(x_{n}\right) \rightarrow y_{0}^{\prime}$ with $y_{0}^{\prime} \in G^{\prime}$ since

$$
\bar{B}\left(f(x), \tau \cdot \delta_{G^{\prime}}(f(x))\right) \subseteq G^{\prime} .
$$

Now that $f$ is a homeomorphism, it follows that $x_{n} \rightarrow f^{-1}\left(y_{0}^{\prime}\right)$ with $f^{-1}\left(y_{0}^{\prime}\right) \in G$. This is contrary to (19) because of $G$ being open.

Hence the statement in Proposition 4.1 is proved.

Proposition 4.2. Let $X, Y, G, G^{\prime}, f, D, D^{\prime}$ be as in Lemma 4.2. Then there exists a point $\omega_{0} \in D$ such that

$$
\left|f\left(\omega_{0}\right)-f(x)\right|>p \cdot \delta_{G^{\prime}}(f(x)) .
$$

Here $p=2 /(3 \eta(2))$.

Proof. If the assertion would not hold, then

$$
|f(y)-f(x)| \leq p \cdot \delta_{G^{\prime}}(f(x))
$$

for all $y \in D$. Since the metric space $X$ is a dense space, it follows that the ring domain $\left\{z \in X: \delta_{G}(x) / 2<|z-x|<\delta_{G}(x)\right\}$ is not a empty set, i.e., $\left\{z \in X: \delta_{G}(x) / 2<\right.$ $\left.|z-x|<\delta_{G}(x)\right\} \neq \emptyset$. Choosing a point $\omega_{1}$ with $\left.\delta_{G}(x) / 2<\left|\omega_{1}-x\right|<\delta_{G}(x)\right\}$, it is evident to see that

$$
\left|f\left(\omega_{1}\right)-f(x)\right| \leq p \cdot \delta_{G^{\prime}}(f(x)) .
$$


Taking $\tau=2 / 3$ in Proposition 4.1, it follows that there exists a point $\omega_{2} \in B\left(x, \delta_{G}(x)\right)$ such that

$$
\left|f\left(\omega_{2}\right)-f(x)\right| \geq(2 / 3) \cdot \delta_{G^{\prime}}(f(x)) .
$$

Thus, combining (20) and (21), we see that

$$
\frac{\left|f\left(\omega_{2}\right)-f(x)\right|}{\left|f\left(\omega_{1}\right)-f(x)\right|} \geq \frac{(2 / 3) \cdot \delta_{G^{\prime}}(f(x))}{p \cdot \delta_{G^{\prime}}(f(x))}=\frac{2}{3 p} .
$$

Moreover, noting that $\left.f\right|_{D}$ is a $\eta$-QS and

$$
\frac{\left|\omega_{2}-x\right|}{\left|\omega_{1}-x\right|}<2
$$

we have

$$
\frac{\left|f\left(\omega_{2}\right)-f(x)\right|}{\left|f\left(\omega_{1}\right)-f(x)\right|} \leq \eta\left(\frac{\left|\omega_{2}-x\right|}{\left|\omega_{1}-x\right|}\right)<\eta(2) .
$$

By comparing (22) with (23), we obtain

$$
p>\frac{2}{3 \eta(2)}
$$

This inequality leads to a contradiction, which completes the proof of Proposition 4.2.

For convenience, we denote by $B^{*}$ an open ball centered at $f(x)$ of radius $\delta_{D^{\prime}}(f(x))$, i.e.,

$$
B^{*}=B\left(f(x), \delta_{D^{\prime}}(f(x))\right) .
$$

Proposition 4.3. Let $X, Y, G, G^{\prime}, f, D, D^{\prime}$ be as in Lemma 4.2. If $\delta_{D^{\prime}}(f(x))<$ $(1 / 2) \cdot \delta_{G^{\prime}}(f(x))$, then

$$
f^{-1}\left(B^{*}\right) \nsubseteq B\left(x, \frac{\delta_{G}(x)}{2}\right)
$$

Proof. If the conclusion was false, then

$$
f^{-1}\left(B^{*}\right) \subseteq B\left(x, \frac{\delta_{G}(x)}{2}\right)
$$

Our first goal is to show that there exists a sequence $\left\{v_{n}\right\}$ in $Y \backslash D^{\prime}$ which has an accumulation point in $G^{\prime}$.

By definition of $\delta_{D^{\prime}}(f(x))$, it is clear that there exists a sequence of points $\left\{v_{n}\right\} \subseteq$ $Y \backslash D^{\prime}$ such that

$$
\delta_{D^{\prime}}(f(x)) \leq\left|v_{n}-f(x)\right|<\delta_{D^{\prime}}(f(x))+\frac{1}{n} .
$$

Noting the assumption $\delta_{D^{\prime}}(f(x))<(1 / 2) \cdot \delta_{G^{\prime}}(f(x))$, it is trivial to see that

$$
\left|v_{n}-f(x)\right|<\delta_{D^{\prime}}(f(x))+\frac{1}{n}<\frac{\delta_{G^{\prime}}(f(x))}{2}+\frac{1}{n}<\frac{3}{4} \delta_{G^{\prime}}(f(x))
$$

for sufficiently large $n$ (i.e., $\left.n>4 / \delta_{G^{\prime}}(f(x))\right)$.

The inequality (25) and the fact $\left\{v_{n}\right\} \subseteq Y \backslash D^{\prime}$ imply that

$$
v_{n} \in G^{\prime} \backslash D^{\prime}
$$

for sufficiently large $n$. Recall that $Y$ is proper, we know that the sequence $\left\{v_{n}\right\}$ has a cluster point in $Y$. For simplicity, we may assume that

$$
v_{n} \rightarrow y^{*}
$$


for some $y^{*} \in Y$ as $n$ goes to infinity. According to (26) and $D^{\prime}$ being open, we have

$$
y^{*} \notin D^{\prime} \text {. }
$$

Furthermore, from the inequality (25), we deduce that

$$
\left|y^{*}-f(x)\right|<\delta_{G^{\prime}}(f(x))
$$

which implies that

$$
y^{*} \in G^{\prime} .
$$

The next thing to do in the proof is to construct a sequence $\left\{z_{m}\right\}$ in $B^{*}$ which converges to the point $y^{*}$. Due to the metric space $Y$ being dense and the inequality (28), it is easy to see that

$$
B\left(y^{*}, \frac{1}{m}\right) \cap B\left(f(x), \delta_{D^{\prime}}(f(x))\right) \neq \emptyset
$$

for each positive integer $m \in \mathbf{N}$. Choosing the points

$$
z_{m} \in B\left(y^{*}, \frac{1}{m}\right) \cap B^{*},
$$

it is obvious that

$$
z_{m} \rightarrow y^{*} \text { as } m \rightarrow \infty
$$

Since $f$ is a homoeomorphism from $G$ onto $G^{\prime}$ and the conclusion (29) holds, we have

$$
f^{-1}\left(z_{m}\right) \rightarrow f^{-1}\left(y^{*}\right) \in G \quad \text { as } \quad m \rightarrow \infty .
$$

We are now in a position to get a contradiction. Combining (24) and (30), it is not difficult to verify that

$$
\left|f^{-1}\left(z_{m}\right)-x\right|<\frac{\delta_{G}(x)}{2}
$$

for each $m \in \mathbf{N}$. Thus, by the above inequality and (31), we get

$$
\left|f^{-1}\left(y^{*}\right)-x\right|<\delta_{G}(x) \text {. }
$$

According to the definition of the domain $D$ as in Lemma 4.2, the above inequality implies that

Consequently, we infer that

$$
f^{-1}\left(y^{*}\right) \in D
$$

$$
y^{*} \in D^{\prime}=f(D)
$$

which contradicts to (27). This completes the proof of Proposition 4.3.

With the help of the preceding three propositions we can now prove Lemma 4.2.

Proof of Lemma 4.2. Evidently, the lemma will be proved if we can show that

$$
\delta_{D^{\prime}}(f(x)) \geq 2 /\left(3 \eta^{2}(2)\right) \cdot \delta_{G^{\prime}}(f(x))
$$

provided that $\delta_{D^{\prime}}(f(x))<(1 / 2) \cdot \delta_{G^{\prime}}(f(x))$. Now, we suppose that

$$
\delta_{D^{\prime}}(f(x))<(1 / 2) \cdot \delta_{G^{\prime}}(f(x)) .
$$

In what follows, we will verify that

$$
\delta_{D^{\prime}}(f(x)) \geq 2 /\left(3 \eta^{2}(2)\right) \cdot \delta_{G^{\prime}}(f(x))
$$

By Proposition 4.2, there exists a point $\omega_{0} \in D$ such that $\left|\omega_{0}-x\right|<\delta_{G}(x)$ and

$$
\left|f\left(\omega_{0}\right)-f(x)\right|>p \cdot \delta_{G^{\prime}}(f(x)) .
$$

Here $p=2 /(3 \eta(2))$. 
According to Proposition 4.3, there exists a point $\omega_{3} \in D$ satisfying

$$
\left|f\left(\omega_{3}\right)-f(x)\right|<\delta_{D^{\prime}}(f(x)) \text { and }\left|\omega_{3}-x\right| \geq \delta_{G}(x) / 2 .
$$

Therefore, from the above statements, it is evident that

$$
\frac{\left|\omega_{0}-x\right|}{\left|\omega_{3}-x\right|} \leq \frac{\delta_{G}(x)}{\delta_{G}(x) / 2}=2 .
$$

Noting that $\left.f\right|_{D}$ is a $\eta$-QS mapping, $\left\{x, \omega_{0}, \omega_{3}\right\} \subseteq D$, and (32) and (33) hold, we obtain that

$$
\frac{p \cdot \delta_{G^{\prime}}(f(x))}{\delta_{D^{\prime}}(f(x))} \leq \frac{\left|f\left(\omega_{0}\right)-f(x)\right|}{\left|f\left(\omega_{3}\right)-f(x)\right|} \leq \eta\left(\frac{\left|\omega_{0}-x\right|}{\left|\omega_{3}-x\right|}\right) \leq \eta(2) .
$$

Thus we have derived that

$$
\delta_{D^{\prime}}(f(x)) \geq \frac{p}{\eta(2)} \delta_{G^{\prime}}(f(x))=\frac{2}{3 \cdot \eta^{2}(2)} \delta_{G^{\prime}}(f(x)),
$$

which completes the proof of Lemma 4.2 .

\section{Proof of the main result}

Proof of Theorem 1.10. Let $x, y \in G$. Denote

$$
B_{x}=B\left(x, \delta_{G}(x)\right) .
$$

In order to prove Theorem 1.10, we need only to show that

$$
\frac{k_{G}(x, y)}{M_{1}} \leq k_{G^{\prime}}(f(x), f(y)) \leq M_{1} k_{G}(x, y) \quad \text { for all } \quad x, y \in G
$$

We divide the proof into two cases:

Case 1. $|x-y| \leq 1 /\left(2 c_{1}\right) \cdot \delta_{G}(x)$. From the definition of $\delta_{G}(x)$, it follows that $x, y \in B_{x}$. Since $\left.f\right|_{B_{x}}$ is $M$-QH, then we have

$$
\frac{k_{B_{x}}(x, y)}{M} \leq k_{f\left(B_{x}\right)}(f(x), f(y)) \leq M k_{B_{x}}(x, y)
$$

Hence, it gives

$$
\begin{aligned}
k_{G^{\prime}}(f(x), f(y)) & \leq k_{f\left(B_{x}\right)}(f(x), f(y)) \quad\left(\text { since } f\left(B_{x}\right) \subseteq G^{\prime}\right) \\
& \leq M \cdot k_{B_{x}}(x, y) \leq 2 M c_{1} \ln \left(1+\frac{|x-y|}{\delta_{G}(x)}\right) \quad \text { (by Lemma 4.1) } \\
& \leq 2 M c_{1} \cdot k_{G}(x, y), \quad \text { (because of the inequality (1)) }
\end{aligned}
$$

which implies the right-hand side inequality of (34).

To prove the left-hand side inequality of (34), we consider two subcases:

Subcase 1.1. $|f(x)-f(y)| \leq 1 /\left(2 c_{2}\right) \cdot \delta_{B_{x}^{\prime}}(f(x))$. From Theorem 3.7, it follows that the map

$$
f^{-1}: D^{\prime}=B\left(f(x), \delta_{B_{x}^{\prime}}(f(x))\right) \rightarrow f^{-1}\left(D^{\prime}\right)=D
$$

is $M_{2}$-Lipschitz in the quasihyperbolic metric, where $M_{2}=16 c_{1} c_{2} M^{2} \cdot \max \left\{c_{1}, c_{2}\right\}$. Since $Y$ is a $c_{2}$-quasiconvex metric space, from Lemma 4.1 and Lemma 4.2 , it follows 
that

$$
\begin{array}{rlr}
k_{G}(x, y) & \leq k_{D}(x, y) \quad(\text { since } D \subseteq G) \\
& \leq M_{2} \cdot k_{D^{\prime}}(f(x), f(y)) \quad\left(\text { since }\left.f^{-1}\right|_{D^{\prime}} \text { is } M_{2} \text {-QH }\right) \\
& \leq 2 M_{2} c_{2} \ln \left(1+\frac{|f(x)-f(y)|}{\delta_{B_{x}^{\prime}}(f(x))}\right) \quad(\text { by Lemma 4.1) } \\
& \leq 2 M_{2} c_{2} \ln \left(1+\frac{|f(x)-f(y)|}{q \cdot \delta_{G^{\prime}}(f(x))}\right) \quad \text { (by Lemma 4.2) } \\
& \leq \frac{2 M_{2} c_{2}}{q} \ln \left(1+\frac{|f(x)-f(y)|}{\delta_{G^{\prime}}(f(x))}\right) \quad \text { (by Bernoulli's inequality) } \\
& \leq \frac{2 M_{2} c_{2}}{q} k_{G^{\prime}}(f(x), f(y)) . \quad \text { (because of the inequality (1)) }
\end{array}
$$

Subcase 1.2. $|f(x)-f(y)|>1 /\left(2 c_{2}\right) \cdot \delta_{B_{x}^{\prime}}(f(x))$. By the inequality (1) and Lemma 4.2, we have

$$
k_{G^{\prime}}(f(x), f(y)) \geq \ln \left(1+\frac{|f(x)-f(y)|}{\delta_{G^{\prime}}(f(x))}\right) \geq \ln \left(1+\frac{q}{2 c_{2}}\right) .
$$

Since $X$ is a $c_{1}$-quasiconvex metric space, it follows that there exists a rectifiable arc $\gamma$ joining $x$ and $y$ in $X$ with length $l(\gamma) \leq c_{1}|x-y|$.

Let $\gamma_{s}:[0, l(\gamma)] \rightarrow \gamma$ be the arc length parametrization of $\gamma$ with $\gamma_{s}(0)=x$. For each $t \in[0, l(\gamma)]$, we have $\left|\gamma_{s}(t)-x\right| \leq l(\gamma)$ and

$$
\delta_{G}(x)=\operatorname{dist}(x, X \backslash G) \leq\left|\gamma_{s}(t)-x\right|+\operatorname{dist}\left(\gamma_{s}(t), X \backslash G\right) \leq t+\delta_{G}\left(\gamma_{s}(t)\right) .
$$

Hence, remembering that $|x-y| \leq \frac{1}{2 c_{1}} \delta_{G}(x)$, (36) implies that

$$
\begin{aligned}
k_{G}(x, y) & \leq \int_{0}^{l(\gamma)} \frac{d t}{\delta_{G}\left(\gamma_{s}(t)\right)} \leq \int_{0}^{l(\gamma)} \frac{d t}{\delta_{G}(x)-t}=\ln \left(1+\frac{l(\gamma)}{\delta_{G}(x)-l(\gamma)}\right) \\
& \leq \ln \left(1+\frac{c_{1}|x-y|}{\delta_{G}(x)-c_{1}|x-y|}\right) \leq \ln 2 .
\end{aligned}
$$

Combing the above with (35), we have

$$
k_{G}(x, y) \leq \frac{\ln 2}{\ln \left(1+q /\left(2 c_{2}\right)\right)} k_{G^{\prime}}(f(x), f(y))
$$

Denote

$$
M_{0}=\max \left\{2 M c_{1}, \frac{2 M_{2} c_{2}}{q}, \frac{\ln 2}{\ln \left(1+q /\left(2 c_{2}\right)\right)}\right\} .
$$

Hence, we get that

$$
\frac{k_{G}(x, y)}{M_{0}} \leq k_{G^{\prime}}(f(x), f(y)) \leq M_{0} k_{G}(x, y) \quad \text { for } \quad|x-y| \leq \frac{1}{2 c_{1}} \delta_{G}(x) .
$$

Case 2. $|x-y|>1 /\left(2 c_{1}\right) \cdot \delta_{G}(x)$. From Theorem 2.7 we know that the metric space $\left(G, k_{G}\right)$ is a 2-quasiconvex metric space. Thus there exists a curve $\gamma:[a, b] \rightarrow G$ joining $x$ and $y$ with quasihyperbolic length

$$
l_{k_{G}}(\gamma) \leq 2 k_{G}(x, y)
$$


Define inductively successive points $x=z_{0}, z_{1}, \cdots, z_{n-1}, z_{n}=y$ of $\gamma$ as follows: Let $t_{0}=a$,

$$
t_{j}=\sup _{t}\left\{t \in[a, b]:\left|\gamma(t)-z_{j-1}\right| \leq \frac{\delta_{G}\left(z_{j-1}\right)}{2 c_{1}}, 1 \leq j \leq n\right\}
$$

and $z_{j}=\gamma\left(t_{j}\right), 0 \leq j \leq n$. Furthermore, since $|x-y|>\left(1 /\left(2 c_{1}\right)\right) \delta_{G}(x)$, we have $n \geq$ $2,\left|z_{j-1}-z_{j}\right|=\left(1 /\left(2 c_{1}\right)\right) \delta_{G}\left(z_{j-1}\right)$ for $1 \leq j \leq n-1$ and $\left|z_{n-1}-z_{n}\right| \leq\left(1 /\left(2 c_{1}\right)\right) \delta_{G}\left(z_{n-1}\right)$.

Hence, by Case 1 , we have

$$
\begin{aligned}
k_{G^{\prime}}(f(x), f(y)) & \leq \sum_{j=1}^{n-1} k_{G^{\prime}}\left(f\left(z_{j-1}\right), f\left(z_{j}\right)\right)+k_{G^{\prime}}\left(f\left(z_{n-1}\right), f(y)\right) \\
& \leq M_{0}\left(\sum_{j=1}^{n-1} k_{G}\left(z_{j-1}, z_{j}\right)+k_{G}\left(z_{n-1}, y\right)\right) \\
& \leq M_{0} \cdot l_{k_{G}}(\gamma) \leq 2 M_{0} \cdot k_{G}(x, y) .
\end{aligned}
$$

Similarly, by using Theorem 2.7 , there exists a curve $\beta^{\prime}:[c, d] \rightarrow G^{\prime}$ joining $f(x)$ and $f(y)$ with quasihyperbolic length

$$
l_{k_{G^{\prime}}}\left(\beta^{\prime}\right) \leq 2 k_{G^{\prime}}(f(x), f(y)) .
$$

Let $\beta=f^{-1} \circ \beta^{\prime}:[c, d] \rightarrow G$. Define inductively successive points $x=p_{0}, p_{1}, \cdots, p_{k-1}$, $p_{m}=y$ of $\beta$ as follows: Let $t_{0}=c$,

$$
t_{j}=\sup _{t}\left\{t \in[c, d]:\left|\beta(t)-p_{j-1}\right| \leq \frac{\delta_{G}\left(p_{j-1}\right)}{2 c_{1}}, 1 \leq j \leq m\right\}
$$

and $p_{j}=\beta\left(t_{j}\right), 0 \leq j \leq m$. Moreover, since $|x-y|>\left(1 /\left(2 c_{1}\right)\right) \delta_{G}(x)$, we have $m \geq 2,\left|p_{j-1}-p_{j}\right|=\left(1 /\left(2 c_{1}\right)\right) \delta_{G}\left(p_{j-1}\right)$ for $1 \leq j \leq m-1$ and $\left|p_{m-1}-p_{m}\right| \leq$ $\left(1 /\left(2 c_{1}\right)\right) \delta_{G}\left(p_{m-1}\right)$.

Hence, Case 1 gives

$$
\begin{aligned}
k_{G}(x, y) & \leq \sum_{j=1}^{m-1} k_{G}\left(p_{j-1}, p_{j}\right)+k_{G}\left(p_{m-1}, y\right) \\
& \leq M_{0}\left(\sum_{j=1}^{m-1} k_{G^{\prime}}\left(f\left(p_{j-1}\right), f\left(p_{j}\right)\right)+k_{G^{\prime}}\left(f\left(p_{m-1}\right), f(y)\right)\right) \\
& \leq M_{0} \cdot l_{k_{G^{\prime}}}\left(\beta^{\prime}\right) \leq 2 M_{0} \cdot k_{G^{\prime}}(f(x), f(y)) .
\end{aligned}
$$

This completes the proof of Theorem 1.10 with $M_{1}=2 M_{0}$.

\section{Appendix}

In this Section, we give the following generalization of Bernoulli's inequality (see [7, p. 34]).

Bernoulli's inequality. Suppose that $\alpha>1$. If $x>-1$, then

$$
(1+x)^{\alpha} \geq 1+\alpha x .
$$

Acknowledgement. The authors wish to express their sincere gratitude to the anonymous referee, whose extremely careful reading of the manuscript led to many clarifications and improvements in the text. We are also grateful to Prof. Xiantao Wang for his help. 


\section{References}

[1] Beurling, A., and L. V. Ahlfors: The boundary correspondence under quasiconformal mappings. - Acta Math. 96, 1956, 125-142.

[2] Heinonen, J., and P. Koskela: Definitions of quasiconformality. - Invent. Math. 120, 1995, 61-79.

[3] Heinonen, J., and P. Koskela: Quasiconformal maps in metric spaces with controlled geometry. - Acta Math. 181, 1998, 1-61.

[4] Heinonen, J., P. Koskela, N. Shanmugalingam, and J. T. Tyson: Sobolev classes of Banach space-valued functions and quasiconformal mappings. - J. Anal. Math. 85, 2001, 87139.

[5] Heinonen, J., and S. Semmes: Thirty-three yes or no questions about mappings, measures, and metrics. - Conform. Geom. Dyn. 1, 1997, 1-12.

[6] Huang, X., and J. Liu: Quasihyperbolic metric and quasisymmetric mappings in metric spaces. - Trans. Amer. Math. Soc. (to appear).

[7] Mitrinović, D.: Analytic inequalities. - Springer-Verlag, Berlin-Heidelberg-New York, 1970.

[8] TukiA, P., and J. VÄIsÄlä: Quasisymmetric embeddings of metric spaces. - Ann. Acad. Sci. Fenn. Math. 5, 1980, 97-114.

[9] Tyson, J.: Quasiconformality and quasisymmetry in metric measure spaces. - Ann. Acad. Sci. Fenn. Math. 23, 1998, 525-548.

[10] Tyson, J.: Metric and geometric quasiconformality in Ahlfors regular Loewner spaces. - Conform. Geom. Dyn. 5, 2001, 21-73.

[11] VÄIsÄLÄ, J.: Lectures on $n$-dimensional quasiconformal mappings. - Lecture Notes in Math. 229, Springer-Verlag, Berlin-New York, 1971.

[12] VÄIsÄLÄ, J.: Quasisymmetric embeddings in Euclidean spaces. - Trans. Amer. Math. Soc. 264, 1981, 191-204.

[13] VÄIsÄlÄ, J.: Quasimöbius maps. - J. Anal. Math. 44, 1984/85, 218-234.

[14] VÄIsÄLÄ, J.: Quasiconformal maps of cylindrical domains. - Acta Math. 162, 1989, 201-225.

[15] VÄısÄLÄ, J.: Free quasiconformality in Banach spaces, I. - Ann. Acad. Sci. Fenn. Math. 15, 1990, 355-379.

[16] VÄıs̈̈LÄ, J.: Free quasiconformality in Banach spaces, II. - Ann. Acad. Sci. Fenn. Math. 16, 1991, 255-310.

[17] VÄIsÄLÄ, J.: Free quasiconformality in Banach spaces, III. - Ann. Acad. Sci. Fenn. Math. 17, 1992, 393-408.

[18] VÄIsÄLÄ, J.: Free quasiconformality in Banach spaces, IV. - Analysis and Topology, 1998, $697-717$.

[19] VÄISÄLÄ, J.: The free quasiworld: freely quasiconformal and related maps in Banach spaces. - In: Quasiconformal geometry and dynamics (Lublin 1996), Banach Center Publ. 48, Polish Academy of Science, Warsaw, 1999, 55-118.

Received 23 December 2014 • Accepted 8 May 2015 